\title{
Quantum effects in a free-standing graphene lattice: Path-integral against classical Monte Carlo simulations
}

\author{
B. G. A. Brito, ${ }^{1,2}$ Ladir Cândido, ${ }^{3}$ G.-Q. Hai, ${ }^{1}$ and F. M. Peeters ${ }^{2}$ \\ ${ }^{1}$ Instituto de Física de São Carlos, Universidade de São Paulo, 13560-970, São Carlos, SP, Brazil \\ ${ }^{2}$ Departement Fysica, Universiteit Antwerpen, Groenenborgerlaan 171, 2020 Antwerpen, Belgium \\ ${ }^{3}$ Instituto de Física, Universidade Federal de Goiás, 74001-970, Goiânia, Go, Brazil \\ (Received 13 May 2015; revised manuscript received 2 September 2015; published 13 November 2015)
}

\begin{abstract}
In order to study quantum effects in a two-dimensional crystal lattice of a free-standing monolayer graphene, we have performed both path-integral Monte Carlo (PIMC) and classical Monte Carlo (MC) simulations for temperatures up to $2000 \mathrm{~K}$. The REBO potential is used for the interatomic interaction. The total energy, interatomic distance, root-mean-square displacement of the atom vibrations, and the free energy of the graphene layer are calculated. The obtained lattice vibrational energy per atom from the classical MC simulation is very close to the energy of a three-dimensional harmonic oscillator $3 k_{B} T$. The PIMC simulation shows that quantum effects due to zero-point vibrations are significant for temperatures $T<1000 \mathrm{~K}$. The quantum contribution to the lattice vibrational energy becomes larger than that of the classical lattice for $T<400 \mathrm{~K}$. The lattice expansion due to the zero-point motion causes an increase of $0.53 \%$ in the lattice parameter. A minimum in the lattice parameter appears at $T \simeq 500 \mathrm{~K}$. Quantum effects on the atomic vibration amplitude of the graphene lattice and its free energy are investigated.
\end{abstract}

DOI: 10.1103/PhysRevB.92.195416

PACS number(s): 65.80.Ck, 65.40.De, 68.65.Pq

\section{INTRODUCTION}

Over the last decades, allotropes of carbon have received much attention in the scientific community due to their promising physical and chemical properties. Graphene is an important example of the carbon allotropes discovered in the last decade [1]. It is currently the most popular studied two-dimensional (2D) material. Although numerous studies have been reported on its peculiar electronic and mechanical properties [2-4], some of its basic properties are not yet fully understood, such as its structural stability and the negative thermal expansion coefficient at room temperature.

The thermal expansion coefficient (TEC) of a graphene monolayer is one of the key quantities that have been measured [5,6], and it is important for applications of graphene in electronic devices. Several theoretical and experimental investigations of the TEC of a graphene layer and its temperature dependence have been reported in the last decade, but large discrepancies exist between the results obtained from different methods. Earlier theoretical studies indicated that the corrugation experienced by the graphene sheet due to the out-of-plane vibrations related to the transversal acoustic phonon modes is responsible for its thermal contraction $[7,8]$. It is expected that the anharmonicity in a two-dimensional crystal such as graphene is important because of an intrinsic bending instability coupled to in-plane stretching modes. Zakharchenko et al. performed a classical Monte Carlo simulation of the finite temperature lattice properties of graphene [9] using the empirical bond order potential LCBOPII [10]. They have shown that graphene is strongly anharmonic due to the soft bending modes yielding strong out-of-plane fluctuations. Very recently, Michel, Costamagna, and Peeters presented a theory of anharmonic phonons in two-dimensional crystals [11]. They presented a systematic study of anharmonic effects in an atomic monolayer crystal with a honeycomb lattice structure using both analytical and numerical lattice dynamical methods. Different theoretical simulations have been done trying to reproduce the temperature dependence of the experimentally measured TEC $[8,9,11-13]$. A very recent study indicated that both anharmonic and quantum effects are important in the understanding of the negative TEC of the graphene monolayer and its temperature dependence [13]. In Ref. [13] the unsymmetrized self-consistent field theory for anharmonic crystals was extended to 2D non-Bravais crystals such as graphene. Quantum corrections were included in the $\hbar$ expansion for the one particle density matrix. The structural and thermodynamic properties of a graphene monolayer were studied with inclusion of anharmonicity and quantum corrections. The obtained results showed that quantum effects are important in the thermodynamic properties of graphene for $T<1000 \mathrm{~K}$. Unfortunately, this theoretical approach breaks down for very low temperature.

On the other hand, earlier studies have shown that the quantum zero-point vibrational motion of carbon atoms influences the structural, thermodynamic, and electronic properties of diamond. In a path-integral Monte Carlo (PIMC) simulation, in which atomic nuclei were treated as quantum particles interacting through a Tersoff-type potential, Herrero and Ramírez [14] found that the quantum zero-point lattice expansion in diamond causes an increase in the lattice parameter of $0.5 \%$ and a decrease in the bulk modulus by $5 \%$. This study also indicated that, at least for materials with light atoms such as carbon, the effects associated with the quantum nature of the atomic nuclei are important for the electronic structure of the material. It has been confirmed recently that it leads to a giant zero-point renormalization $(615 \mathrm{meV})$ of the band gap of bulk diamond $[15,16]$.

In order to investigate quantum effects on the mechanical and thermodynamic properties of graphene, we have performed PIMC simulations of some basic structural and thermodynamic quantities of a graphene monolayer at finite temperature. The PIMC is one of the most powerful methods that is able to simulate a quantum system at finite temperature [17]. Our simulation shows that the quantum effects are 
significant for temperatures $T<1000 \mathrm{~K}$. We find an important contribution of the zero-point energy to the lattice vibration and to the expansion of the in-plane interatomic distance in graphene monolayer even at room temperature. In order to evidence the quantum effects in this system, we compare the obtained results from our PIMC calculations against those from classical Monte Carlo simulations.

A variety of atomistic potentials have been used to investigate the thermodynamic and mechanical properties of graphene [18-21]. Recently, Magnin et al. have performed systematically classical Monte Carlo simulations of the thermal expansion coefficient of free-standing graphene using various atomistic potentials [21] that are appropriate to $s p^{2}$ carbon in order to classify among those potentials which compares best to existing reference data from experiments or alternate theories. The potentials covered empirical to semiempirical bond-order types, as well as a model based on tight-binding theory. Among the potentials which are successful in dealing with carbon and hydrocarbon systems is the empirical bondorder (REBO) potential [19,20]. This potential is used to describe a great variety of systems and chemical reactions. In the present paper we treated the carbon atoms in graphene as quantum particles interacting through a REBO potential.

This paper is organized as follows. In Sec. II, we give a shot description of the PIMC formalism. In Sec. III we present the results for the total energy, vibrational energy, the distance between neighbor atoms, mean-square displacement, and free energy as a function of temperature. Finally, we summarize our work in Sec. IV.

\section{COMPUTATIONAL METHODS AND DETAILS}

We performed both PIMC and classical MC simulations for a free-standing graphene monolayer in the temperature range $50-2000 \mathrm{~K}$ and at zero pressure. In the PIMC simulation, the carbon atoms of graphene are considered as quantum particles with a mass of $12.0 \mathrm{amu}$. Each quantum particle is described by a set of $M$ beads forming a cyclic chain or a "polymer." The REBO potential is used to describe the interaction between the carbon atoms with the parameters taken from Refs. [19] and [20]. The PIMC method [22] is based on evaluating the quantum density matrix

$$
\rho\left(R_{0}, R_{M} ; \beta\right)=\int d \mathbf{R}_{1} \ldots d \mathbf{R}_{M-1} \exp \left[-\sum_{m=1}^{M} S^{m}\right]
$$

by sampling the set of paths, $\left\{\mathbf{R}_{0}, \mathbf{R}_{1}, \ldots \mathbf{R}_{M-1}, \mathbf{R}_{M}\right\}$, with $\mathbf{R}_{k}$ being the set of beads $\left\{\mathbf{r}_{1, k}, \ldots, \mathbf{r}_{n, k}\right\}$ and $\mathbf{r}_{i, k}$ a bead which is the position of the $i$ th particle in the $k$ th time slice and $\tau=\beta / M$ with $\beta=1 / k_{B} T$. The action $S^{m} \equiv S\left(\mathbf{R}_{M-1}, \mathbf{R}_{M}\right) \equiv$ $-\ln \left[\rho\left(R_{M-1}, R_{M}\right)\right]$ is handled in the primitive approximation. To evaluate the $3 N M$-dimensional integrals (with $N$ being the number of atoms and $M$ the number of beads) in Eq. (1), we employ the Metropolis bisection sampling technique [17].

The PIMC simulations have been performed in a threedimensional (3D) box using a honeycomb unit cell with periodic boundary conditions in the $x y$ plane, and the atoms are allowed to move in the $z$ direction. Most calculations are performed within a simulation box containing 200 spinless carbon atoms (or $10 \times 10$ unit cells). A "time step" of $\tau=0.00022 \mathrm{~K}^{-1}$ (corresponding to $M=15$ at $T=300 \mathrm{~K}$ ) is used for the discretized imaginary time path integrals. The extrapolation to the $\tau=0$ limit shows that this approximation induces an error of about $0.07 \%$ in the total energy. Checks for larger systems showed that the obtained results do not change within the statistical error when compared to a system with 200 atoms. For the averages we use about 50000 PIMC time step.

\section{RESULTS AND DISCUSSION}

We have first calculated the total internal energy per atom of the two-dimensional crystal as a function of temperature both using quantum and classical MC simulations. This energy can be written as $E(T)=E_{0}+E_{\mathrm{vib}}(T)$, where $E_{0}$ is the cohesive energy for the classical crystal at $T=0$, and $E_{\mathrm{vib}}(T)$ is the vibrational energy which is temperature dependent. By fitting the classical $\mathrm{MC}$ results for the total energy, we obtained $E_{0}=$ $-7.3951 \mathrm{eV}$ with the REBO potential employed here, which is consistent with the result for the cohesive energy $(-7.395 \mathrm{eV})$ obtained by Lindsay and Broido [23] for the graphene crystal with the same potential. Figure 1 gives the obtained energy as a function of temperature in which we have taken zero as the cohesive energy $E_{0}$ of the classical crystal. We see that the classical and quantum MC results approach each other at high temperatures as expected. However, a considerable difference between them is found at low temperatures. Figure 1 shows clearly that quantum effects become significant in the vibrational energy of the graphene layer for temperatures

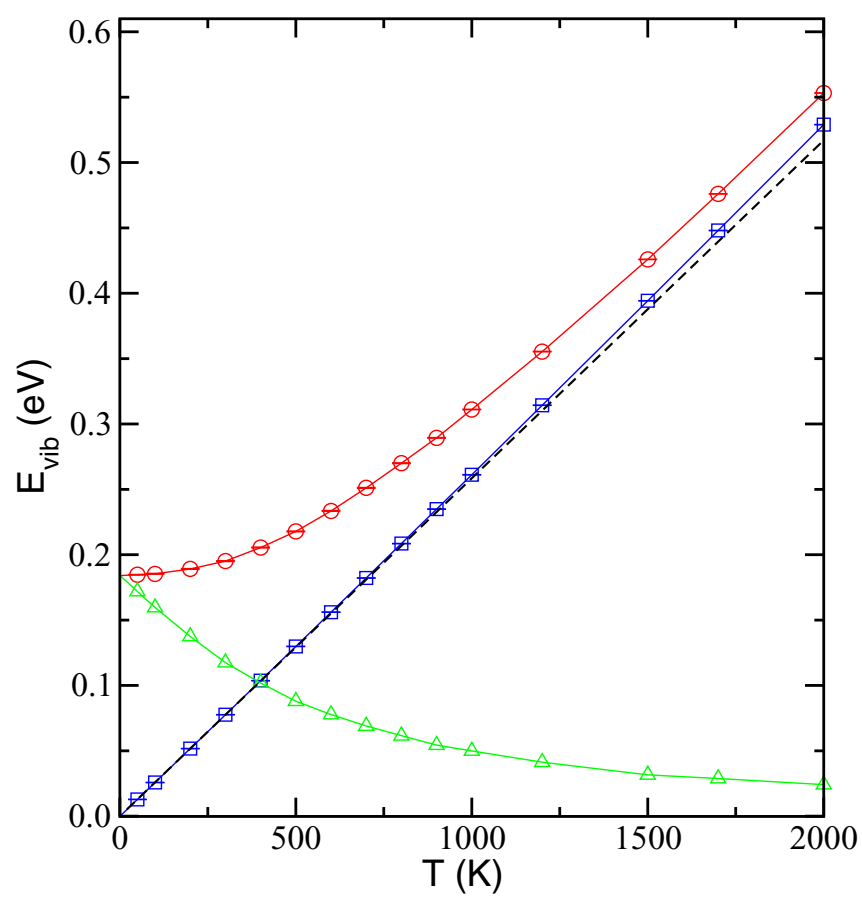

FIG. 1. (Color online) The vibrational energy per atom of the graphene monolayer as a function of temperature at zero pressure. The red circles and blue squares are the PIMC and classical MC results, respectively. The green triangles are the difference between the PIMC and MC results. The black dashed line is the energy of a $3 \mathrm{D}$ harmonic oscillator $3 k_{B} T$. 
$T<1000 \mathrm{~K}$. The classical MC result $E_{\text {vib }}$ is very close to a straight line passing through the origin, while the PIMC result approaches a finite value in the low-temperature limit due to quantum effects, i.e., the zero-point energy. The difference between the quantum and classical MC vibrational energy is given in the figure by the green triangles. This energy difference is of pure quantum origin. For $T \lesssim 400 \mathrm{~K}$, this quantum contribution is larger than the vibrational energy of the classical crystal. In Fig. 1 we also plot by the black-dashed line the total energy of a $3 \mathrm{D}$ harmonic oscillator $3 k_{B} T$ which is very close to the classical MC vibrational energy. Their difference is $0.015 \mathrm{eV}$ (about 3\% only) at $T=2000 \mathrm{~K}$. This means that within the REBO interaction potential the vibrational energy of the free-standing 2D graphene crystal (when it is considered as a classical crystal) is basically given by that of a 3D harmonic oscillator.

Another quantity which is influenced by strong quantum effects is the in-plane distance between the carbon atoms or the lattice parameter $a$. In Fig. 2(a) we show the lattice parameter $a$ (i.e., the in-plane distance between the atoms of the second nearest neighbors) from both the quantum and classical MC simulations. The classical MC results from the REBO potential are consistent with those found in previous work by Magnin et al. [21]. It increases almost linearly with increasing temperature. However, the PIMC simulation shows that the distance between the atoms in a graphene layer has a very different behavior as a function of temperature. The zero-point lattice expansion in the graphene layer causes an increase in the lattice parameter of $0.53 \%$. Its value in the zero-temperature limit obtained from the PIMC calculation is $2.4734 \AA$ against $2.4602 \AA$ from the classical MC simulation. With increasing temperature from zero, the PIMC simulation shows that the lattice parameter decreases to a minimum value of $a=2.4715 \AA$ at $T \approx 500 \mathrm{~K}$, beyond which it increases with increasing temperature. We have also calculated the interatomic distance $a_{1}$ between the atoms of the nearest neighbors which are shown in Fig. 2(b). The obtained interatomic distances $a$ and $a_{1}$ from PIMC simulations have a very similar temperature dependent behavior with a minimum at about $500 \mathrm{~K}$. The ratio of their values is basically equal to $\sqrt{3}$ within less than $0.1 \%$. The value of $a /\left(\sqrt{3} a_{1}\right)$ from PIMC calculations as a function of temperature is given in the inset of Fig. 2(a). The PIMC results of the interatomic distances $a$ and $a_{1}$ are fitted using a fifth-order polynomial $\left(c_{0}+c_{1} T+\right.$ $\ldots+c_{5} T^{5}$ ) given by the red solid curves in the figure. The coefficients of the corresponding polynomials are given by $c_{0}=2.4734 \AA, c_{1}=-5.5889 \times 10^{-6} \AA / \mathrm{K}, c_{2}=-1.7526 \times$ $10^{-9} \AA / \mathrm{K}^{2}, c_{3}=1.5503 \times 10^{-11} \AA / \mathrm{K}^{3}, c_{4}=-1.0739 \times$ $10^{-14} \AA / \mathrm{K}^{4}$, and $c_{5}=2.3255 \times 10^{-18} \AA / \mathrm{K}^{5}$ for the lattice parameter $a$ and $c_{0}=1.4284 \AA, c_{1}=-1.7329 \times 10^{-6} \AA / \mathrm{K}$, $c_{2}=-5.5316 \times 10^{-9} \AA / \mathrm{K}^{2}, c_{3}=1.524 \times 10^{-11} \AA / \mathrm{K}^{3}, c_{4}=$ $-9.7212 \times 10^{-15} \AA / \mathrm{K}^{4}$, and $c_{5}=2.0378 \times 10^{-18} \AA / \mathrm{K}^{5}$ for the interatomic distance $a_{1}$ between the atoms of the nearest neighbors. The thermal expansion coefficient $\alpha$ of the graphene layer can be estimated from the derivative $(1 / a) d a / d T$ [and $\left.\left(1 / a_{1}\right) d a_{1} / d T\right]$ and is given by the solid (dashed) curve in the inset in Fig. 2(b). The derivatives are obtained from the fitted curves of the interatomic distances in Figs. 2(a) and 2(b). Notice that the estimated $\alpha$ in the inset obtained from $a$ and $a_{1}$ are very close to each other.


FIG. 2. (Color online) Temperature dependence of the in-plane interatomic distances in the graphene monolayer between the atoms of (a) the second and (b) the first nearest neighbors obtained from the PIMC (red circles) and classical MC (blue squares) simulations. The red curves are a fifth-order polynomial fitting of the PIMC results. The inset in (a) gives the ratio of the interatomic distances of the second to the first nearest neighbors divided by $\sqrt{3}$, and the inset in (b) shows the thermal expansion coefficient obtained from the derivatives of the fitted interatomic distances of the second (solid curve) and first (dashed curve) nearest neighbors from the PIMC simulations.

When we compare the obtained PIMC results of the lattice vibrational energy and lattice parameter from our calculations with those of diamond obtained by Herrero and Ramírez (Figs. 3 and 8) in Ref. [14], we see that quantum effects 


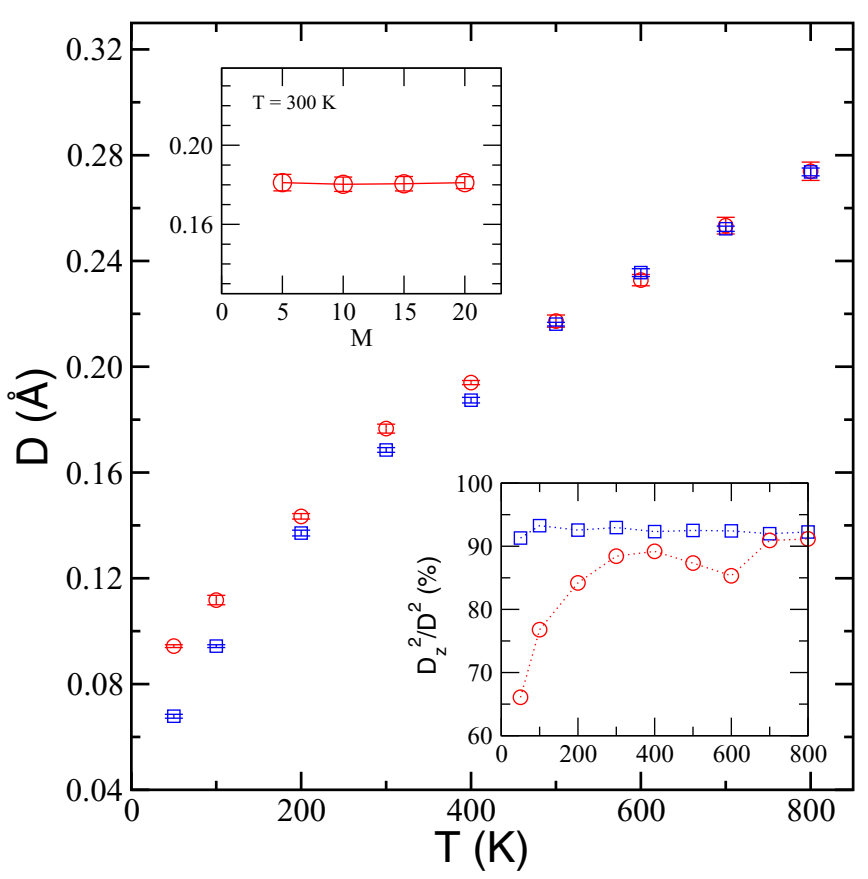

FIG. 3. (Color online) The rms displacement $D$ of the carbon atoms in the graphene layer as a function of temperature. The lower inset shows the relative contribution from the $z$ direction to the total mean-square displacement. The upper inset shows the convergence of $D$ on the number of beads $M$ in the PIMC simulation for $T=300 \mathrm{~K}$. The red circles and blue squares are the results obtained from PIMC and classical MC simulations, respectively.

are significant at low temperature both in 2D graphene and 3D diamond lattices. The zero-point motion of the carbon atoms lead to a lattice parameter expansion of about $0.5 \%$ at $T=0 \mathrm{~K}$ in both systems. But an important difference is that quantum effect pushes the minimum of the lattice parameter in $2 \mathrm{D}$ graphene to $T \simeq 500 \mathrm{~K}$ which does not happen in $3 \mathrm{D}$ diamond. In diamond, the minimum lattice parameter is at $T=0 \mathrm{~K}$. This may contribute to the origin of the negative thermal expansion coefficient of graphene at low temperatures $(T \lesssim 500 \mathrm{~K})$.

From the PIMC simulations, we also estimate the displacement of the atoms from their equilibrium position at finite temperature due to thermal vibrations. The mean-square displacement $D^{2}$ of a quantum particle is given by [24]

$$
D^{2}=\frac{1}{M}\left\langle\sum_{i=1}^{M}\left(\mathbf{r}_{i}-\langle\overline{\mathbf{r}}\rangle\right)^{2}\right\rangle
$$

where $\langle\cdots\rangle$ indicates a thermal average at temperature $T$ and $\overline{\mathbf{r}}$ is the centroid of the "polymer."

The root-mean-square (rms) displacement $D$ of the carbon atoms in the graphene layer obtained both from the classical and quantum MC simulations are given in Fig. 3. The upper inset shows the convergence of the rms displacement on the number of beads $M$ in the PIMC simulation. At low temperatures the PIMC results of the rms displacement are slightly larger than those obtained from our classical MC simulations. We also confirm that the contribution to the mean-square displacement from the movement of the carbon atoms in the $z$ direction (perpendicular to the graphene layer) is much larger than that in the $x y$ plane $[25,26]$. This is shown in the lower inset of Fig. 3 where the contribution from the movement of the atoms in the $z$ direction $D_{z}$ to the total mean-square displacement $D$ in the graphene layer is plotted. It indicates that, when the atoms are considered as classical particles, the thermal vibrations in the $z$ direction contribute more than $90 \%$ of the total mean-square displacement. When the carbon atoms are treated as quantum particles, the PIMC calculation shows that such a contribution decreases at low temperatures $(T \lesssim 300 \mathrm{~K})$, and the relative contribution in the $x y$ plane $D_{x y}$ to the total mean-square displacement increases. It means that the quantum movement of the carbon atoms in the $x y$ plane in the graphene layer is of importance for its temperature dependent properties such as the vibrational energy and the interatomic distances for temperatures below room temperature.

We can write $D^{2}$ as resulting from contributions from the following two terms,

$$
D^{2}=\frac{1}{M}\left\langle\sum_{i=1}^{M}\left(\mathbf{r}_{i}-\overline{\mathbf{r}}\right)^{2}\right\rangle+\left\langle(\overline{\mathbf{r}}-\langle\overline{\mathbf{r}}\rangle)^{2}\right\rangle=D_{q}^{2}+D_{c}^{2} .
$$

The first term $D_{q}^{2}$ in the above expression corresponds to the average spatial extension of the paths of the quantum particles, and the second term $D_{c}^{2}$ gives the mean-square displacement of the centroid. In the high-temperature limit, only the second term remains because each path collapses onto a single point.

As the atomic vibration in the graphene layer is anisotropic, we need to analyze separately the rms displacement of the atoms in the $z$ direction and in the $x y$ plane. The PIMC calculation shows that the rms displacement in the $z$ direction $D_{z}$ is basically determined by the centroid displacement $D_{c, z}$, i.e., the classical movement is dominant in the $z$ direction. Such behavior is expected because the graphene layer is free standing in this direction. In the $x y$ plane, the rms displacement $D_{x y}$ is mainly determined by $D_{c, x y}$ for $T \gtrsim 1000 \mathrm{~K}$. But different behavior is observed at lower temperatures where $D_{q, x y}$ becomes significant, as is shown in Fig. 4. This happens because in the $x y$ plane the carbon atoms are being subjected to a strong potential due to the covalent bond of graphene, and quantum effects from the zero-point lattice vibration become significant at low temperatures. For $T \lesssim 250 \mathrm{~K}$ the radius of gyration of the paths gives a larger contribution to the in-plane rms displacement. The enhancement of the quantum delocalization of the atoms in the $x y$ plane at low temperatures is consistent with the quantum lattice expansion observed above.

We finally compute the free energy of the graphene layer using the adiabatic switching (AS) [27] and reversible scaling (RS) [28] methods. The AS method is an efficient alternative for the high computational cost involved in the standard thermodynamic integration method [29]. Its efficiency comes from the coupling parameter varying continuously during the simulation and the integration along the thermodynamic path being performed dynamically. Although the AS method is very efficient, it provides only the free energy at a single temperature. For problems that require the knowledge of the free energy in a certain range of temperatures, several simulations are needed. The RS method allows us to obtain the free energy for a large interval of temperatures. To apply 


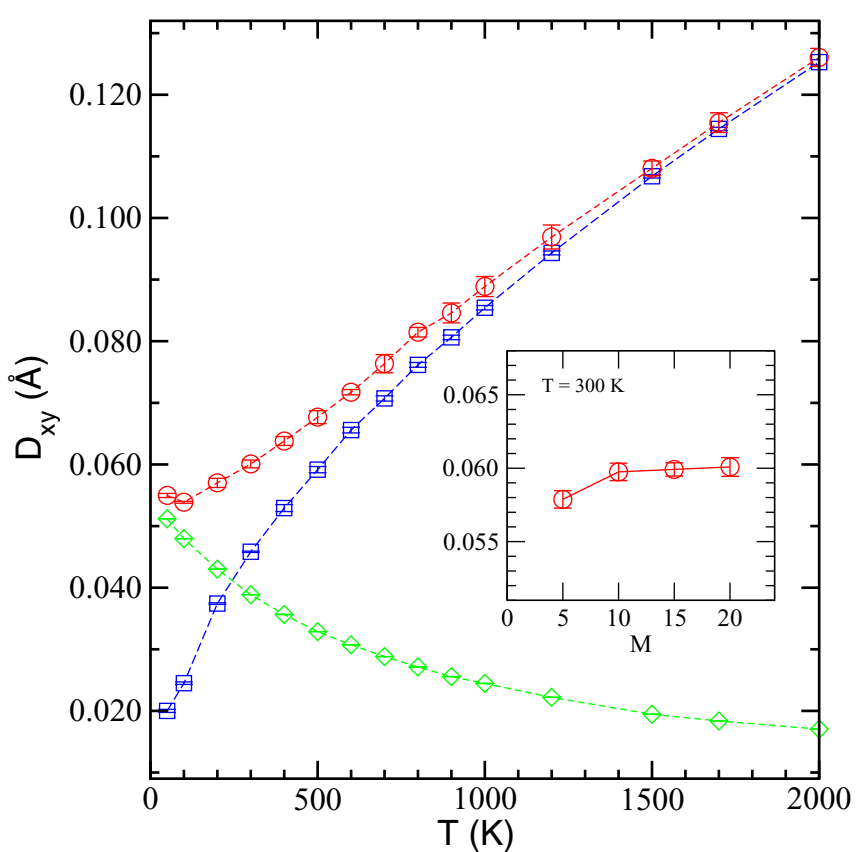

FIG. 4. (Color online) The PIMC results of the in-plane rms displacement $D_{x y}$ (red circles) as a function of temperature. The rms displacement of the centroid $D_{c, x y}$ (blue squares) and the rms radius-of-gyration $D_{q, x y}$ (green diamonds) of the quantum paths are also given. The inset shows the dependence of $D_{x y}$ on the number of beads $M$ for $T=300 \mathrm{~K}$.

this method we just need the previous knowledge of the value of free energy for one temperature which can be obtained using the AS method. The extension of these methods to the path integral formalism can be found in Refs. [30] and [31].

We have calculated the free energy of the system using the AS method. This method requires a reference system whose Helmholtz free energy is known in advance. Normally the Einstein crystal is used as a reference for the solid phase. The Einstein crystal is a collection of harmonic oscillators with the mass of the carbon atom. In the temperature range under consideration, we choose an anisotropic Einstein crystal with in-plane oscillation frequency $\omega_{1}=300 \mathrm{~cm}^{-1}$ and frequency $\omega_{2}=100 \mathrm{~cm}^{-1}$ for the oscillations in the perpendicular direction. We have used about $10^{5}$ Monte Carlo steps for the calculation of the difference of Helmholtz free energy between the one of the graphene layer and the Einstein crystal both in the classi-

TABLE I. The classical and quantum MC results of the free energy obtained from the adiabatic switching (AS) method at different temperatures. The digits in parentheses are estimated standard errors in the last decimal places.

\begin{tabular}{lll}
\hline \hline & \multicolumn{2}{c}{ Free Energy (eV) } \\
\cline { 2 - 3 } $\mathrm{T}(\mathrm{K})$ & $\mathrm{MC}$ & \multicolumn{1}{c}{ PIMC } \\
\hline 200 & $-7.3030(13)$ & $-7.2105(11)$ \\
600 & $-7.2894(11)$ & $-7.2466(11)$ \\
1200 & $-7.4014(8)$ & $-7.3792(6)$ \\
2000 & $-7.6736(13)$ & $-7.6607(12)$ \\
\hline \hline
\end{tabular}

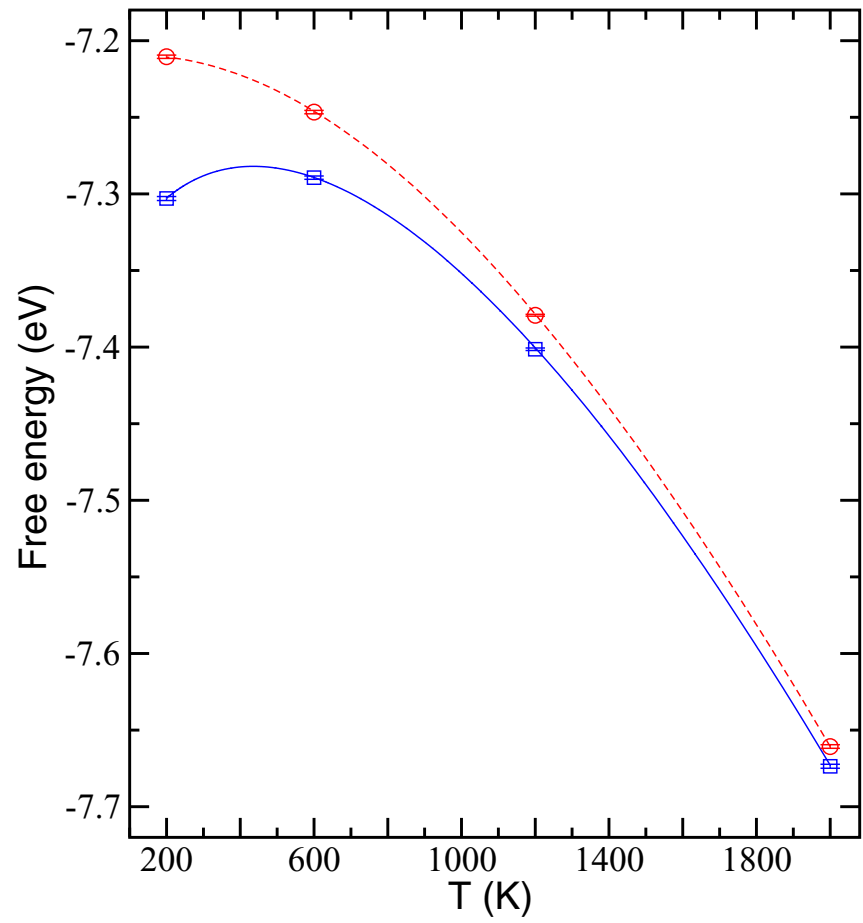

FIG. 5. (Color online) Free energy of the free-standing graphene monolayer. The results obtained from the AS method are given by the red circles and blue squares for the PIMC and classical MC simulations, respectively. The red and blue curves are the corresponding results from the RS method.

cal and quantum simulations. The obtained free energies from the AS method at different temperatures are given in Table I.

Using the free energy estimated by the AS method at $T=2000 \mathrm{~K}$, we performed simulations in the range of $9 \times 10^{6}$ MC steps within the NPT ensemble using the RS method in order to determine the temperature dependence of the free energy. In Fig. 5, we show the results of the free energy obtained from the RS method in the temperature range from 200 to $2000 \mathrm{~K}$ for both classical and quantum Monte Carlo simulations, together with the AS results given in Table I. The difference between AS and RS results are within the error bar indicated in Table I. As expected, similar convergent behavior at high temperature is observed between the classical and quantum MC results as found in the total energy calculation. Figure 5 also shows that with decreasing temperature the free energy from the classical MC simulation deviates from the quantum results. For $T \lesssim 400 \mathrm{~K}$ the classical calculation yields unphysical behavior for the free energy, showing a negative variation of the system entropy. This unphysical behavior points clearly to the importance of quantum effects in the graphene layer at low temperatures.

\section{CONCLUSION}

We have performed both classical and path-integral MC simulations in order to study quantum effects on the structural and thermodynamic properties of a free-standing graphene monolayer. The same REBO potential is used to describe the interatomic interaction. The obtained PIMC results at low 
temperatures are very different from those of the classical simulation. The quantum effects of zero-point vibration are significant for temperatures up to $1000 \mathrm{~K}$, and the quantum contribution to the lattice vibrational energy becomes larger than that of the classical lattice for $T<400 \mathrm{~K}$. The zero-point lattice expansion in the graphene layer causes an increase in the lattice parameter of $0.53 \%$ leading to a minimum of the lattice parameter at $T \simeq 500 \mathrm{~K}$ and, consequently, a negative thermal expansion coefficient for $T<500 \mathrm{~K}$. We also analyzed quantum effects on the atomic vibration amplitude of the graphene lattice and its free energy. The free energy of the graphene layer is calculated in the temperature range 200-2000 K.
The temperature dependence of the free energy shows that the classical MC simulation cannot describe correctly the free energy of the system for $T<400 \mathrm{~K}$ indicating once more the importance of quantum effects on the thermodynamic properties of the free-standing graphene layer.

\section{ACKNOWLEDGMENTS}

This research was supported by the Brazilian agencies FAPESP, FAPEG, and CNPq, the Flemish Science Foundation (FWO-Vl) and the Methusalem Foundation of the Flemish Government.
[1] K. S. Novoselov, A. K. Geim, S. V. Morozov, D. Jang, Y. Zang, S. V. Dubonos, I. V. Grigorieva, and A. A. Firsov, Science 306, 666 (2004).

[2] A. H. Castro Neto, F. Guinea, N. M. R. Peres, K. S. Novoselov, and A. K. Geim, Rev. Mod. Phys. 81, 109 (2009).

[3] C. Józsa, M. Popinciuc, N. Tombros, H. T. Jonkma, and B. J. van Wees, Phys. Rev. Lett. 100, 236603 (2008).

[4] D. Li and R. B. Kaner, Science 320, 1170 (2008).

[5] D. Yoon, Y-W. Son, and H. Cheong, Nano Lett. 11, 3227 (2011).

[6] W. Bao, F. Miao, Z. Chen, H. Zang, W. Jang, C. Dames, and C. N. Lau, Nat. Nanotechnol. 4, 562 (2009).

[7] A. Fasolino, J. H. Los, and M. I. Katsnelson, Nat. Mater. 6, 858 (2007).

[8] N. Mounet and N. Marzari, Phys. Rev. B 71, 205214 (2005).

[9] K. V. Zakharchenko, M. I. Katsnelson, and A. Fasolino, Phys. Rev. Lett. 102, 046808 (2009).

[10] J. H. Los, L. M. Ghiringhelli, E. J. Meijer, and A. Fasolino, Phys. Rev. B 72, 214102 (2005).

[11] K. H. Michel, S. Costamagna, and F. M. Peeters, Phys. Rev. B 91, 134302 (2015).

[12] J.-W. Jiang, J.-S. Wang, and B. Li, Phys. Rev. B 80, 205429 (2009).

[13] A. L. C. da Silva, L. Cândido, J. N. Teixeira Rabelo, G.-Q. Hai, and F. M. Peeters, Europhys. Lett. 107, 56004 (2014).

[14] C. P. Herrero and R. Ramírez, Phys. Rev. B 63, 024103 (2000).

[15] F. Giustino, S. G. Louie, and M. L. Cohen, Phys. Rev. Lett. 105, 265501 (2010).

[16] E. Cannuccia and A. Marini, Phys. Rev. Lett. 107, 255501 (2011).

[17] D. M. Ceperley, Rev. Mod. Phys. 67, 279 (1995).
[18] F. H. Stillinger and T. A. Weber, Phys. Rev. B 31, 5262 (1985); J. Tersoff, ibid. 37, 6991 (1988); J. Che, T. Cagin, and Goddard, III, Theor. Chem. Acc. 102, 346 (1999); J. H. Los and A. Fasolino, Comput. Phys. Commun. 147, 178 (2002); Phys. Rev. B 68, 024107 (2003).

[19] D. W. Brenner, Phys. Rev. B 42, 9458 (1990).

[20] D. W. Brenner, O. A. Shenderova, J. A. Harrison, S. J. Stuart, B. Mi, and S. B. Sinnott, J. Phys.: Condens. Matter 14, 783 (2002).

[21] Y. Magnin, G. D. Forster, F. Rabilloud, F. Calvo, A. Zappelli, and C. Bichara, J. Phys.: Condens. Matter 26, 185401 (2014).

[22] E. L. Pollock and D. M. Ceperley, Phys. Rev. B 30, 2555 (1984).

[23] L. Lindsay and D. A. Broido, Phys. Rev. B 81, 205441 (2010).

[24] M. J. Gillan, Philos. Mag. A 58, 257 (1988).

[25] J. H. Los, M. I. Katsnelson, O. V. Yazyev, K. V. Zakharchenko, and A. Fasolino, Phys. Rev. B 80, 121405(R) (2009); S. Chen and D. C. Chrzan, 84, 195409 (2011).

[26] P. Xu, M. Neek-Amal, S. D. Barber, J. K. Schoelz, M. L. Ackerman, P. M. Thibado, A. Sadeghi, and F. M. Peeters, Nat. Commun. 5, 3720 (2014).

[27] M. Watanabe and W. P. Reinhardt, Phys. Rev. Lett. 65, 3301 (1990).

[28] M. de Koning, A. Antonelli, and S. Yip, Phys. Rev. Lett. 83, 3973 (1999).

[29] D. Frenkel and B. Smit, Understanding Molecular Simulation (Academic, San Diego, 1996).

[30] R. Ramírez, C. P. Herrero, A. Antonelli, and E. R. Hernández, J. Chem. Phys. 129, 064110 (2008).

[31] B. G. A. Brito and A. Antonelli, J. Chem. Phys. 137, 034114 (2012). 